\title{
Research Strategies for Biomedical and Health Informatics
}

\section{Some Thought-provoking and Critical Proposals to Encourage Scientific Debate on the Nature of Good Research in Medical Informatics}

\author{
Reinhold Haux1; Casimir A. Kulikowski; Suzanne Bakken ${ }^{3}$; Simon de Lusignan4; \\ Michio Kimura5; Sabine Koch ${ }^{6}$; John Mantas7; Victor Maojo ${ }^{8}$; Michael Marschollek ${ }^{1}$; \\ Fernando Martin-Sanchez ${ }^{9}$; Anne Moen ${ }^{10,11}$; Hyeoun-Ae Park ${ }^{12}$; Indra Neil Sarkar ${ }^{13}$; \\ Tze Yun Leong ${ }^{14,15}$; Alexa T. McCray ${ }^{16}$
}

${ }^{1}$ Peter L. Reichertz Institute for Medical Informatics, University of Braunschweig and Hannover Medical School, Germany; ${ }^{2}$ Department of Computer Science, Rutgers - The State University of New Jersey, NJ, USA;

${ }^{3}$ School of Nursing and Department of Biomedical Informatics, Columbia University, New York, NY, USA:

${ }^{4}$ Department of Clinical and Experimental Medicine, University of Surrey, Guildford, UK;

${ }^{5}$ Medical Informatics Department, School of Medicine, Hamamatsu University, Shizuoka, Japan;

${ }^{6}$ Department of Learning, Informatics, Management and Ethics, Health Informatics Centre, Karolinska Institutet,

Stockholm, Sweden:

${ }^{7}$ Health Informatics Laboratory, National and Kapodistrian University of Athens, Athens, Greece;

${ }^{8}$ Biomedical Informatics Group, Artificial Intelligence Department, Universidad Politecnica de Madrid, Madrid, Spain;

${ }^{9}$ Department of Healthcare Policy and Research, Division of Health Informatics, Weill Cornell Medicine, New York,

NY, USA;

${ }^{10}$ Institute for Health and Society, Faculty of Medicine, University of Oslo, Oslo, Norway;

${ }^{11}$ Institute for Health Sciences, University College of South East Norway, Drammen, Norway;

${ }^{12}$ College of Nursing and Systems Biomedical Informatics Research Center, Seoul National University, Seoul, Republic of Korea;

${ }^{13}$ Center for Biomedical Informatics, Brown University, Providence, RI, USA;

${ }^{14}$ Medical Computing Laboratory, School of Computing, National University of Singapore, Singapore;

${ }^{15}$ School of Information Systems, Singapore Management University, Singapore;

${ }^{16}$ Department of Biomedical Informatics, Harvard Medical School, Boston, MA, USA

\section{Keywords}

Biomedical informatics, health informatics, medical informatics, research, education

\section{Summary}

Background: Medical informatics, or biomedical and health informatics (BMHI), has become an established scientific discipline. In all such disciplines there is a certain inertia to persist in focusing on well-established research areas and to hold on to well-known research methodologies rather than adopting new ones, which may be more appropriate.

Correspondence to:

Prof. Dr. Reinhold Haux

Peter L. Reichertz Institute for Medical Informatics

University of Braunschweig - Institute of Technology

and Hannover Medical School

Muehlenpfordtstr. 23

38106 Braunschweig

Germany

E-mail: reinhold.haux@plri.de
Objectives: To search for answers to the following questions: What are research fields in informatics, which are not being currently adequately addressed, and which methodological approaches might be insufficiently used? Do we know about reasons? What could be consequences of change for research and for education?

Methods: Outstanding informatics scientists were invited to three panel sessions on this topic in leading international conferences (MIE 2015, Medinfo 2015, HEC 2016) in order to get their answers to these questions.

Methods Inf Med 2017;56(Open): e1-e10 https://doi.org/10.3414/ME16-01-0125 received: November 5, 2016 accepted: November 17, 2016 published: January 25, 2017
Results: A variety of themes emerged in the set of answers provided by the panellists. Some panellists took the theoretical foundations of the field for granted, while several questioned whether the field was actually grounded in a strong theoretical foundation. Panellists proposed a range of suggestions for new or improved approaches, methodologies, and techniques to enhance the BMHI research agenda.

Conclusions: The field of BMHI is on the one hand maturing as an academic community and intellectual endeavour. On the other hand vendor-supplied solutions may be too readily and uncritically accepted in health care practice. There is a high chance that $\mathrm{BMHI}$ will continue to flourish as an important discipline; its innovative interventions might then reach the original objectives of advancing science and improving health care outcomes. 


\section{Introduction}

Medical informatics, or biomedical and health informatics (BMHI), has become an established scientific discipline (e.g $[1,2,3$, $4,5,6])$. Through its international and national associations (as, e.g., documented in $[7,8])$, and through its scientific conferences, journals and periodicals (e.g. [9]) researchers have been communicating their research results and contributing to scientific progress for more than five decades. By hopefully maintaining high scientific and ethical standards in research and in reviewing and selecting research papers for publication, they thereby share new knowledge, mainly by presenting original research articles and systematic reviews on a wide range of research topics.

Medical informatics has a clear objective. As a discipline, "concerned with the systematic organization, representation, and analysis of data, information and knowledge in biomedicine and health care" it "aims to contribute to high-quality, efficient health care and to quality of life on the one hand and to progress in science on the other" [10].

In all disciplines there is a certain inertia in keeping to well-established research areas (for major research areas in medical informatics see e.g. $[11,12])$ to persist, even as they suffer from diminishing impact. Likewise, there is frequently a tendency for researchers of a discipline to hold on to well-known research methodologies or technologies rather than adopting new methodological or technical approaches that may contribute more effectively to research outcomes and advance collective and interdisciplinary understanding (see e.g. [13, 14, 15]). These observations prompt a number of questions:

1. What are highly original and relevant research fields for BMHI, which are not adequately addressed by current medical informatics research?

2. Which methodological or technical approaches are insufficiently used in trying to solve highly original and relevant research questions in our field?

3. Do we know about or can we guess at reasons for the above?

4. What could be consequences of change for research strategies?
5. What could be consequences of change on the knowledge and skills that have to be included in educational programs and courses in BMHI (e.g. [16, 17])?

To discuss this matter and to provide answers to the five questions, panel sessions entitled "Are we doing the right research in biomedical and health informatics and are we doing it right?" have been organized at Medical Informatics Europe (MIE) 2015 in Madrid, Spain [18], at Medinfo 2015 in Sao Paulo, Brazil [19], and at the Health Exploring Complexity Conference 2016 (incorporating MIE 2016) in Munich, Germany [20].

As organiser of the panels the first author of this manuscript (RH) invited outstanding informatics scientists and moderated the panel sessions at these leading international conferences. In addition to their long-term scientific activities most of the panel participants are or have been presidents or board members of leading informatics organizations with international impact such as IMIA [7], APAMI [21], EFMI [8], ACMI [22], and AMIA [23].

Twelve of the fourteen panellists (SB, SdeL, MK, SK, CK, JM, VM, MM, FMS, AM, HAP, and INS) agreed to document their updated panel contributions in this paper. Their responses can be found in chapters one to five. Finally the last two authors (TYL and ATM) summarized the panellists' answers.

It is our hope that some maybe critical and provocative thoughts can be presented in order to contribute to the scientific debate on the nature of good research in BMHI and its impact on the future of our field - a theme, which always has been an important tradition in this journal (see e.g. $[1,23]$, and, more recently, [24] with [25] and $[26,27]$ with $[28]$ and $[29,30]$ with [31] and [32], as well as [33] with [34] and [35]). We are now putting them up for discussion and invite readers to send us their comments and their thoughts, preferably as letters to the editor.

On the authors' contributions for this manuscript: RH is responsible for the introduction and for the overall organization, TYL and ATM for the discussion chapter. Each panellist contributed to this paper by providing their individual viewpoints on the five questions. All authors also contributed to the introduction, in particular $\mathrm{CK}$, who was involved from the beginning with these panels together with $\mathrm{RH}$.

In the following chapters one to five each panellist's individual viewpoint (in alphabetic order) forms one section in this chapter. So the respective viewpoints can be clearly assigned to each panellist.

\section{What are highly original and relevant research fields for biomedical and health informatics, which are not adequately ad- dressed by current medical informatics research?}

Suzanne Bakken: My contention is that discovery and intervention research in BMHI often lack a solid theoretical foundation although a theoretical base is a defining characteristic of a scientific discipline.

Simon de Lusignan: Linking phenotype - as defined within computerised medical records, especially the high quality data in sentinel networks $[36,37]$ to (1) 'combined-omics data' (genomics, proteomics, metabolomics): There is so much potential to answer and generate research questions; e.g. extending our understanding of diabetes [38]; (2) big data: We make very little use of unstructured/free text (written or audio); or make questionnaires part of clinical data; or add biometric data - from generic smart phones, as well as specific sensors. Better use of imaging for diagnosis and treatment. There is much potential for low cost imaging to improve care, e.g. ultrasound; whereas current practice favours high cost, high quality imaging systems. The latter are often relatively inaccessible.

Michio Kimura: I started my career in engineering faculty before going to medical school. There, I constructed a clinical counselling system as a master's dissertation [39]. A pressure tank manufacturer wanted to apply knowledge-based systems to the design of a high pressure tank [40]. At the factory, I was shown many pictures 
of fractured tanks. The engineers there explained them as their 'gems', saying that "Fractured tank cases are precious, as we learn much from failures, which costed highly.". We can tell many other cases. A travel bag company provides their products free to airline crews, and replaces them by a new one, provided that they sent back the broken ones. By this, a company learns which part is likely to be broken. A baseball team manager says "Some wins are reasonless, but there is no reasonless loss." [41]. After graduation from medical school, I found there are only few failure reports in medical science. I find only few ' $\mathrm{xxx}$ is not effective to disease yyy.' reports. Same things happen in basic science fields, too. There seems to be a large difference in sense of values between scientists and engineers. The former being 'discovering significant rules, theses, findings first' while the latter has 'developed or invented techniques used by many people. The economic value of thoughts and invention is protected by other disciplines than intellectual rights. At least, clinical medicine should seek the latter, while accepting the intellectual right protection. Thus medical informatics, especially in applied systems, should follow this [42].

Sabine Koch: With the advent of new sources of health data we observe some current trends. Clinical research is going towards personalised and precision medicine. Health care providers are eager to measure the effect of their actions and to build a learning health care system. Data science is the new buzzword in computer science. Patients start measuring themselves and analyse their own data to personalise their treatment.

Casimir Kulikowski: Interpreting heterogeneous models of biomedical information for scientific inquiry and experimental design is needed across the spectrum of health and disease [43], based on neuroscience, cognitive-perceptual, social and utilitarian/economic considerations [44, 45] with explanation and prediction at multiple scales (nano-to-population, [46]); resolutions (as in imaging); and levels of abstraction with the implicit metaphorical assumptions behind idealized mathematical models [47]. For health care practices, we need theories of social interaction and role-dependent human 'gaming' under risk and uncertainty with responsibilities and accountabilities in ethics and law [48], based on cultural and microbiome interactions [49] and with cognitive linguistics theories of narrative, and underlying semantics of conceptual blending [50]. Sensory iconic representations for interface and graphical schemas [51] need to be developed so as to capture the tradeoffs between expressive visual languages and their ambiguities, with the goal of moving towards better cognitive models of individual normative care.

John Mantas: The field of BMHI is both theoretical and practical. In recent years the domain has been overwhelmed by the applications side; hence the term eHealth is dominant and most of the conferences are focused on that. Being that, the dominant area of the evidence-based and the evaluation of applications play an increased role. However, if we have to reflect on our field we have to think about the principles of setting up a research question. We usually have the following types of research questions, for example: (a) exploratory questions, such as "What are the specifications of a system?", (b) experimental questions, such as "Is prediction according to a given theory?", (c) theoretical questions, such as "What theory explains the observed data?".

Victor Maojo: Considering science, topics that (1) address basic scientific issues, focused on fundamental, informational problems (which may lead to Nobel Prizes). E.g., information theories in biology/chemistry, such as information coding, processing and transmission in the brain, can lead to paradigm shifts in neurology and psychiatry. And there are topics that (2) go beyond the current BMHI scope. E.g., nanomedicine, a promising new field for medicine, and its associated 'nanoinformatics' area. Last, but not least (3) early artificial intelligence work in decisionmaking and knowledge modeling should be revisited. Considering engineering, to investigate cognitive and usability issues related to BMHI. E.g., more intuitive, visual interfaces. Similarly, to increase early training to facilitate use of BMHI tools, beginning at medical schools. Also, systems for improving voice recognition for computer data entry. From a strategic perspective, a
'Hilbert-like' agenda [52] for envisioning fundamental research in the next decades might be necessary.

Michael Marschollek: Regarding the three - still up-to-date - goals set in the 2013 prognosis paper by Haux et al., patient-centered recording and use of data for cooperative care, process-integrated decision support and comprehensive use of patient data for research [53], we still underachieve to some extent. We are faced with an ever increasing amount of new data from new data sources - patient and device-generated - and struggle to integrate and analyze these along with other clinical data, because of technical difficulties, imprecise semantics, but also because of doubts as to their quality and trustworthiness. Industrial players freely use such data to provide health related advice, with little or no quality control, creating a huge service-oriented health 'apps' business. In clinical informatics, we have not achieved broad-scale semantic interoperability, a prerequisite for ambitious decision support as well as for interdisciplinary secondary use research.

Fernando Martin-Sanchez: Exposome Informatics - Environmental health informatics, processing individual-level environmental risk data and their integration with genome and phenome data will hugely impact biomedical research and the discovery of underlying causes of diseases [54]. Participatory Health Informatics Consumer-generated health data through participatory technologies (mobile health, social media, self-quantification, direct to consumer services) will play a vital role on prevention [33]. Health Informatics in Virtual Worlds - Technological developments based on (mobile) augmented reality and virtual reality (e.g. Pokemon GO) are opening up a myriad of opportunities for health informatics with potential impact on education, training and patient activation [55].

Anne Moen: We should ask more questions to scrutinise our assumptions, and therefore ask questions 'if - when - how' standardized and formalized representations play out in fine-tuned, socio-cultural webs of personal, professional, organizational, technical and culturally oriented health and wellness activities [56]. Furthermore, I would like to see more co-creating re- 
search-based practice models for personalized care based on (a) interdisciplinary insights, (b) collaboration, coordination and continuity in and across care trajectories, and (c) digital health literacy.

Hyeoun-Ae Park: Most data in health care are unstructured. Sources of such unstructured data include free-text narratives in the electronic health records or postings in social media. In order to analyze unstructured data, especially social big data, researchers put a lot of efforts and time to collect terms and structuralize them. However, the terms collected and structures developed tend to be managed in a silo. So there is lack of reusability and interoperability from one project to the other. Based on this observation, I would like to pick lack of framework (such as ontology or data models) for unstructured social big data analytics as one of research fields not adequately addressed by current medical informatics research.

Indra Neil Sarkar: Biomedical informatics has evolved into a discipline that has its own theories and methodologies, but we must remain focused on our rich history of developing practical applications for clinicians and patients that address realworld clinical needs. Second, we must emphasize the fostering of purposeful team science approaches that lead to the development of trans-disciplinary teams. Finally, we must enforce the improvement of standards instead of creating a new to enable focus on improving interoperability.

\section{Which methodological or technical approaches are insufficiently used in trying to solve highly orig- inal and relevant research questions in our field?}

Suzanne Bakken: As a discipline with an applied component, BMHI needs development and testing of middle range theories as strategy for knowledge building and advancing the field as a scientific discipline. Grand theories are broad in scope, contain general concepts and propositions, and are typically not testable via empirical methods. A number of grand theories have in- formed BMHI research: general systems theory [57], Shannon-Weaver model of communication [58], human problem solving [59]. In addition, Blois explicated a grand theory for BMHI in his seminal work on the nature of information in medicine [60]. In contrast to grand theories, middle range theories are narrower in scope, have fewer concepts, and are testable [61]. In "When conversation is better than computation", Coiera explicated a middle range theory based on the notion of the communication-information continuum [62]. The scope of the middle range theory is the health care organizational communication space. He argues that developing a richer understanding of communication tasks should help to design and implement information and communication technologies to meet organizational information needs. The middle range theory has informed multiple studies including those by Collins et al. about communication and documentation in the intensive care unit $[63,64,65]$.

Simon de Lusignan: More use is needed of real world evidence. This should complement not substitute for randomized controlled trials. We need to understand what constitutes good quality real world evidence (from computerized medical records) and when best to use. Maybe long term follow up of trial participants [66]. Challenge of how to incorporate technology into clinical workflow; the barriers are more than just usability [67].

Michio Kimura: Evaluation of cases of not only reported ones, but all cases of real population. We have electronic medical records, which record all patient cases.

Sabine Koch: Informatics approaches to tackle new research questions stay however largely the same. We structure the data, try to make it semantically interoperable and of high quality, and build frameworks to interpret it.

Casimir Kulikowski: Integration of language, image, speech and recorded actions is needed to define new paradigms for experimental design in both the science and the clinical practice of BMHI.

John Mantas: Therefore, given our basis on the data, information, and knowledge model of the health care domain we are facing with research questions that are ex- ploratory, theoretical and experimental: that is, observations are made of a system hence data are collected which can be processed to receive information, a theory is proposed which explains those observations, new predictions are made from this theory, and experiments are conducted to verify the predictions.

Victor Maojo: More research on hypothesis generation and problem solving. BMHI still lacks outstanding results from a scientific perspective. From the 20 most cited BMHI papers in the ThomsonReuters' ISI Web of Science, only a few might be labelled as 'scientific'. Some papers actually suggest that some keywords can predict a higher probability of acceptance in some BMHI-related journals [68], which may indicate that highly original research or papers may well end up negatively peer-reviewed while some papers which just follow a current trend may have a higher chance of being accepted.

Michael Marschollek: We need to understand and teach more advanced (temporal) data analytics methods, likely leading to hybrid models combining medical domain knowledge and machine learned content.

Fernando Martin-Sanchez: Research should always rest on "methodologies that capture the processes integral to applications, the users and the world in which the users function" [69]. If we are to generate new knowledge in health informatics, the methodology used in a study must show how concepts from health sciences are integrated with concepts from information sciences. So far, exemplars of these integrative conceptual models or frameworks have been sporadic [70]. Instead, there have been many attempts to implement conceptual models from information and communication technologies in the health sector, with little success in many cases.

Anne Moen: Develop methodologies for sound and rigorous analysis of media rich data; e.g., real time images, video or unstructured text, to explore patient effort as they compare or contrast their own health data to people similar to me, and to see more research seeking to elicit real clinicians' robust, operating strategies in our everyday health practices. 
Hyeoun-Ae Park: Ontology development is one of the technical approaches insufficiently used to solve social big data analytics problems. If we would like to derive insights and contextual meaning from social big data, we need to have an ontology - explicit formal specifications of the terms in the domain and relations among them [71] - as a framework for social big data analytics. Developing an ontology includes: defining classes in the ontology, arranging the classes in a taxonomic (subclass - superclass) hierarchy, defining slots and describing allowed values for these slots, and filling in the values for slots for instances [72].

Indra Neil Sarkar: Innovations in biomedical informatics must be grounded in the realities of health care. Therefore, input from patients and true clinical stakeholders (not those who know informatics) is essential. Alongside descriptions of methodological innovations for understanding disease or impact care, there must be requirement of open data sets and open source code to accompany publications (akin to molecular sequence data).

\section{Do we know about or can we guess at reasons for the above?}

Suzanne Bakken: I believe that the major reason for this is that many education programs emphasize the engineering rather than scientific aspects of the discipline. There are multiple consequences of this lack. First, discoveries may lack context; this could worsen in the era of big data. Second, the active ingredients of BMHI interventions may be unknown and difficult to replicate. Third, inadequate theoretical foundations in BMHI research will result in failure to build knowledge and advance the field as a scientific discipline.

Simon de Lusignan: Lack of serious career progression/opportunities particularly within clinical medicine. Part of the problem is the failure to define our discipline [73] and its sub-disciplines and their core theory [74].

Michio Kimura: Reluctance of reporting non-positive results, though there are many learnable failures.
Sabine Koch: Multi-disciplinarity is both the challenge and the opportunity of our field. We borrow methods and theories from many different fields and the definition of genuine BMHI methods and theories is difficult. Whereas we can find medical informatics methods such as e.g. signal processing or knowledge representation, the theoretical and conceptual base of our field is rather blurry. Another characteristic is the smooth transition between research and application. Per definition medical informatics aims "to contribute to high-quality, efficient health care and to quality of life on the one hand and to progress in science on the other" [10]. We have to admit that we failed in achieving this aim. Many informatics applications are not adapted to the users' needs and thus not used or not used as intended, which prevents the expected benefits from being achieved.

Casimir Kulikowski: The field tends to accept the technologies of vendors with minimal questioning or change in current practices. New, responsible models for cyber-interactions must involve embodied cognition [75] with language/speech and visual/sensory interactions to help us escape the 'prison of abstract data' we are currently in so as to move towards more realistic ways of personalizing and individualizing health care.

John Mantas: Unfortunately, most of the research is conducted not to the whole spectrum described above but only at particular stages. Reasons for that is that the research is focused mainly on the applications either to develop them or to evaluate them, hence missing the main goal which is that research is required to verify the theory or to develop new theory, therefore, to increase the knowledge of the field of biomedical informatics and not simply to evaluate applications, which may be very useful but should not be our only main goal.

Victor Maojo: Currently, there might be a proliferation of topics such as useracceptance, statistical analysis (particularly correlation-based, as in big data) and ontological issues in published papers, sometimes overlooking causation, scientific explanations and long-term impact.

Michael Marschollek: The simple answer is that moving and ever-changing targets are difficult to hit, and that we are often caught in our routine. A more thought-provoking answer may be that we are driven by research agendas, that we incline to do as we were taught or told, that we rather run with the pack and may over time become 'comfortably numb'.

Fernando Martin-Sanchez: We have been suffering the effects of a scientific/ academic system that punishes interdisciplinarity (academic careers, grants, publications, conferences). As a result of this, many academic groups and centers show some inertia in the way they conduct research. The current education model also contributes to this resistance to change. It has been recognized that the most exciting developments in science are taking place at the interfaces between different areas of knowledge. Addressing informatics challenges around the concepts of exposure science, participatory health and virtual worlds represents a good example.

Anne Moen: My explanations would be in the direction that there are (a) weak conventions for trustworthiness, representativeness and quality in studies using media rich data, and (b) 'pilotitis', i.e. studies do not scale nor transfer across practice systems, settings or countries.

Hyeoun-Ae Park: Developing an ontology for a specific domain requires a considerable time because it involves collecting terms for class, slots and values; establishing hierarchical relationship among classes and adding additional details on each class. Thus, researchers tend to use speedy vocabulary management for social big data analytics.

Indra Neil Sarkar: The maturity of informatics has led to limited incentive to share our ideas with the community of beneficiaries (e.g., clinicians). More crosscommunication between clinical and patient oriented publication venues with informatics ones is needed. Academic culture also encourages self-promotion.

\section{What could be consequences of change for research strategies?}

Suzanne Bakken: The impact of developing middle range theories is an increase in the knowledge base for the discipline. 
Simon de Lusignan: International/PanEuropean training around agreed principles and theory that define our discipline, rather than local service specification [76].

Michio Kimura: Clinical medicine of course started to learn from errors. Incidents/accidents are reported in welcoming atmosphere, for the purpose of discarding learnable errors. Then, how about research studies? Clinical trials became mandated to register the protocol, before deployment [77]. This, however, is not only by the above motivation, mainly for patient rights protection, as we still see very few negative result reports. We are happy to see a journal with technical reports and negative reports. Unlike commercial companies, researchers are allowed to fail. It is allowed because failures are reported in learnable manner.

Sabine Koch: We need to strengthen the theoretical and conceptual base of our field. This may be done by adapting existing theories if necessary. We further need to bridge better between the theory and practice of biomedical informatics. Participatory design approaches involving different professionals and different types of users, including patients, need to be adapted in broader scale when developing informatics applications.

Casimir Kulikowski: Inquiry for science and care-giving informatics for practice ought to consider neurosciencebehavioral-economic models which take into account affective as well as utilitarian factors in both selfish goal-driven and altruistic behaviors $[78,79]$. BMHI is the only professional discipline modeling the entire scope of human behavior not strictly based on self-centric assumptions.

John Mantas: We can understand this weakness in our research as we have not fully formalized the theoretical basis of our field as other scientific or engineering disciplines have already done. The health care domain requiring quite understandably practical results for the benefit of the patient enhances the tendency in our field to pursue the applications side using clinical trials or epidemiological approaches for applications evaluations, whereas the theoretical part of our domain based on information processing background is gradually shifting to a lower priority.
Victor Maojo: Medical industry actually develops hundreds of informatics applications without using BMHI results and professionals. With thousands of medical apps in the market, will BMHI be finally necessary at all for industry (and science)? As a consequence, a shift in governmental support based on financial considerations (e.g., in current agencies' open strategy and BMHI support for training and research), could have a dramatic impact on BMHI. Finally, patients will always be there to be treated, but will there still be well-trained biomedical informaticians - as we know them today?

Michael Marschollek: To avoid grinding to a halt, we should more frequently leave the beaten tracks with a small group of enthusiastic researchers and try risky, unconventional approaches, not fearing repeated failures, because from those we learn the most.

Fernando Martin-Sanchez: Making a greater emphasis on designing, developing, implementing and evaluating specific integrative conceptual models for health informatics would contribute to raise the profile of our discipline, increasing our scientific recognition and placing us in a better position to interact with other disciplines.

Anne Moen: Bringing the clinical professionals back to the community, to produce contributions based on a needed re-turn to clinical practice, appreciating situatedness and context-bound data information - knowledge to understand variation and strategies to personalize professional judgment or relevant personal health activities.

Hyeoun-Ae Park: If an ontology is used as a framework for a social big data analytics, it will be helpful in deriving insights, in understanding keyword relationships, in obtaining further details on each term, and in getting contextual meaning of each term in a particular domain.

Indra Neil Sarkar: Identify ways to collaborate (not compete) with related disciplines, including those underpinning data science (e.g., computer science, biostatistics, epidemiology). Develop common repositories or journal policies to encourage open use. Host 'hackathons' to demonstrate value of open data.
6 . What could be consequences of change on the knowledge and skills that have to be included in educational programs and courses in biomedical and health informatics?

Suzanne Bakken: The addition of middle range theories requires a change in BMHI educational programs and courses. Some authors have proposed general competencies in theories. For example, Kulikowski et al. proposed the understanding and application of "syntactic, semantic, cognitive, social, and pragmatic theories as they are used in biomedical informatics" [17]. However, such competencies do not emphasize the development and testing of middle range theories for BMHI research.

Simon de Lusignan: Yes, we need skills and modules around core informatics theory and practice in all clinical training pathways [80]. However, the post-graduate pathway that should produce this disciplines new academic leaders is the most lacking.

Michio Kimura: Analyses based on all cases of electronic medical records. Data reconciliation methods to make databases for all populations from scattered databases of providers. Structured evaluation methods of failures, as well as successes.

Sabine Koch: We do not only train students devoted to research but many who will work in health care settings and industry. These students need to know which genuine biomedical informatics methods or theories from other fields are available, how and under which circumstances to apply them, and which effects to expect from their application.

Casimir Kulikowski: Stimulating new ways of scientific thinking and dispassionate assessment of professional practices in socio-economic and political environments and contexts. Human-technological ecosystems centered on individual care, rather than ethically-constrained or compromised group or population-centered care alone needs to be emphasized.

John Mantas: The adoption of the IMIA educational recommendations [16] is a 
good sign that we can both balance the theoretical and applications contents of our field at adequate level. Depending on the focus of postgraduate courses either on research or on applications, curriculum contents are usually modified, accordingly. Curricula should be adapted and updated continuously to fit the local needs as well as the upcoming technologies without losing the baseline of the theoretical background of the discipline.

Victor Maojo: Health analytics (and big data) is hardly differentiated from classical statistical areas. This trend, generally labelled as 'data science', can eliminate the borders between BMHI and other fields, as it actually happens in some BMHI programs. Instruction should be adapted to each profile (e.g., nanomedicine and nanotechnology, for nanoinformatics). Master of science and $\mathrm{PhD}$ programs must encourage independent and original scientific thinking while some current programs may actually emphasize professional profiles rather than scientific.

Michael Marschollek: We should aim to establish a cross-fertilizing relationship between students and teachers driven by enthusiasm for the ultimate and noble goal of medical informatics - improvement of health for all of us - which is fundamental, though not easy to achieve. In medical students, we need to spark interest or at least establish understanding for our discipline, ideally by involving them actively in our research projects.

Fernando Martin-Sanchez: The next generation of health informaticians needs to learn how to work with integrative conceptual models and apply them when implementing biomedical research and clinical solutions. This will make them more aware of the full potential of research on health informatics.

Anne Moen: Educational offerings should include discussions of inherent ambiguity in clinical information, logics of professional work or personal care activities, and interdisciplinary contributions beyond ones primary frame of reference, knowledge or expertise.

Hyeoun-Ae Park: For ontology development, students need to have fundamental knowledge on health care, personal health and population health to under- stand the domain where an ontology is being developed. In addition, natural language processing and semantic technologies are required as one of fundamental knowledge under technological approach. However, knowledge representation - a method of encoding concepts and relationships in a domain using definitions that are computable - is among the most important skills needed to be emphasized in educational programs.

Indra Neil Sarkar: We must embrace team science approaches that integrate informatics students with health students. Additionally, there needs to be design of curricula that focus on applied clinical informatics and interface with basic informatics research.

\section{Discussion}

A variety of themes emerged in the set of answers provided by the panellists. Some panellists took the theoretical foundations of the field for granted, while several questioned whether the field was actually grounded in a strong theoretical foundation. Panellists proposed a range of suggestions for new or improved approaches, methodologies, and techniques to enhance the BMHI research agenda. They offered opinions on the reasons for the failure to fully adopt some of these approaches, as well as the potential positive consequences of integrating these new or improved approaches. Finally, the panellists proposed some changes that would need to be made to BMHI training programs if the field were to evolve in the suggested ways.

The main research themes identified include: fundamental theories of BMHI, personalized medicine in context, and a research agenda driven by real world problems.

Many argued that the BMHI field lacked a solid theoretical foundation both for discovery and intervention research. This means that discoveries, for example, through data mining methods, were likely to lack context. Further, without a solid foundation, informatics interventions for health care might be difficult to replicate. Perhaps, just as importantly, without a theoretical foundation it is extremely diffi- cult to build the necessary knowledge to advance the field as a scientific discipline. One panellist proposed that the field adopts the principles of the middle range theory [81]. Middle range theories are distinguished from grand theories and are narrower in scope. A middle range theory aims to integrate theory and empirical research by abstracting away from empirical data to create testable, verifiable hypotheses. Another panellist suggested that basic research in the field needs to operate at multiple scales, resolutions, and levels of abstraction, and that research in health care practice would do well to adopt theories of social interaction and gaming and multiple agent decision and action systems.

Indeed, advances in personalized medicine cannot be achieved in specific, isolated situations or narrow settings. Some panellists argued that causal discoveries and optimal interventions should be conducted through the continuum of care trajectory from personal health, community and social wellness, through clinical care and population. Others highlighted that scientific investigations and innovative solutions should effectively combine the different -omic perspectives of the individuals, carefully mitigate the complex interactions between the human and the environment, and systematically address the interdisciplinary challenges in socio-economical and political contexts.

Several panellists advocated that the future research agenda should be driven by real world problems, with deep engagement and input from patients and clinical stakeholders. Such investigations, while aiming for practical impact, should focus on the fundamental and applied research issues, instead of simply developing or evaluating applications.

According to many of the panellists, the research agenda in the BMHI field could be advanced by adopting new and improved approaches, methodologies, and techniques. Broad based, integrative approaches are needed to support full cycles of exploratory, theoretical, and experimental investigations. In some cases these will result from collaboration with scientists in adjacent fields thereby strengthening the interdisciplinary nature of the field. New discoveries and disruptions often happen 
at the interfaces among different areas of knowledge. New paradigms for experimental design and evaluation are needed, and predictions based on the existing data-toinformation-to-knowledge paradigm need to be verified. Many insights can be gained from working with real world data from different perspectives, and analysis and understanding of both successful and failed cases. Additional modalities such as language, image, speech, and multimedia need to be incorporated. Temporal data approaches are needed, and more real world evidence, including data from large-scale electronic health record systems and longitudinal data clinical trials are also needed.

One pressing area is to pursue better methods for integrating heterogeneous data from multiple sources, while ensuring that the quality and trustworthiness of the data are assessed. An important research area continues to be the development and adoption of data standards. One panellist suggested that a framework for the field should be developed based on ontologies and data models for unstructured data, while another panellist cautioned that it would be important to assess the impact of formal representations on representing a range of health care activities. High on the list of additional data types to incorporate in biomedical and health information research are -omics data, including genomic, proteomic, metabolomics and environmental data with the goal of integrating these with clinical data. Approaches for incorporating data generated from mobile health, social media, and a range of biometric applications need to be explored and developed. The emerging focus on precision medicine and personalized care has great potential for the further development of the BMHI field.

One panellist suggested possible reasons why some of the above-mentioned approaches may not have been readily adopted. These included that researchers tend to conduct research driven by (perhaps) externally imposed research agendas; that they continue to use techniques that they learned as students; and that they tend to use conventional research techniques because these are widely used and accepted. Another panellist suggested that, in fact, there is a publication bias to publish re- search that follows established trends, rather than publish highly original research that perhaps has taken methodological risks. Interdisciplinary approaches are a challenge because methodological boundaries are possibly blurred, and academic systems may not be prepared to effectively evaluate such research. On the other hand, one panellist argued that it is important to identify ways to collaborate with rather than compete with related fields, and another argued that a greater emphasis on integrative, interdisciplinary models for BMHI would likely raise the profile of the field. Publications that include open data sets and source code are available to a much broader audience and would, in theory, lead to greater cross-fertilization of ideas among researchers and disciplines.

Panellists proposed some changes that will need to be made to BMHI training programs given the suggestions they have made for improvements to the informatics research agenda. The existing IMIA educational recommendations [16] will need to be adapted and updated regularly. The theoretical underpinnings of middle range theory, if adopted, will need to be taught. New ways of scientific thinking should be stimulated, and students should be encouraged to pursue original scientific approaches. Some panellists argued that digital health literacy and how to apply or develop the right informatics tools and systems to conduct research should be taught to all medical students; these should complement the rigorous training in professional practices in different socioeconomic and political environments or contexts. It will continue to be important to acknowledge the broad range of career trajectories for informatics students some of whom will be pursuing careers in academia, while others will work in health care settings, or in industry. Students should learn to work with integrative models in their research, and they should be exposed to research beyond their primary frames of reference, knowledge, and expertise. Team science approaches that bring informatics students together with students in the health care disciplines should be promoted. The challenges and the importance of supporting patient care through the continuum should be highlighted.
The field of biomedical and health informatics is at a crossroads. We are maturing as an academic and intellectual community, but our recent research has largely focused on specific, small-scale application projects and evaluation studies. Many panellists reckon that our profession is at risk of being marginalized. Some highlighted the worrisome trends that many informatics tools in use are developed without informatics professionals. Also, most medical and health care professionals just readily accept the vendor supplied solutions and technologies in their routine work. If the proposed changes in research directions are adopted, there is a high chance that the new advancements would raise the profile of the field, and influence a shift in government support for health care from one that is solely based on financial considerations. This will allow BMHI to continue to flourish as an important discipline, with many innovative interventions to reach the original objectives of advancing science and improving health care outcomes.

\section{References}

1. van Bemmel JH. Medical informatics, art or science? Methods Inf Med. 1996; 35: 157-172; discussion 173-201.

2. Musen MA, van Bemmel JH. Challenges for medical informatics as an academic discipline. Methods Inf Med. 2002; 41: 1-3.

3. Kulikowski CA. IMIA: coalescing medical informatics worldwide for 40 years. Yearb Med Inform. 2007: 176-185. Erratum in: Yearb Med Inform. 2008: 19.

4. Haux R. Medical informatics: past, present, future. Int J Med Inform. 2010; 79: 599-610.

5. Shortliffe EH. The future of biomedical informatics: a perspective from academia. Stud Health Technol Inform. 2012; 180: 19-24.

6. Lehmann CU, Jaulent MC, Séroussi B, editors. IMIA yearbook of medical informatics 2016. Special 25th anniversary edition. Stuttgart: Schattauer; 2016.

7. International Medical Informatics Association [cited 2016 Sept 25]. Available from: http://imiamedinfo.org.

8. European Federation for Medical Informatics [cited 2016 Sept 25]. Available from: http://www. efmi.org.

9. Maojo V, Garcia-Remesal M, Bielza C, Crespo J, Perez-Rey D, Kulikowski C. Biomedical informatics publications: a global perspective. Part I: Conferences. Methods Inf Med. 2012; 51: 82-90 and Part II: Journals. Methods Inf Med. 2012; 51: 131-137. 
10. Haux R. On determining factors for good research in biomedical and health informatics. Some lessons learned. Yearb Med Inform. 2014; 9: 255-264, discussion 265-272.

11. Schuemie MJ, Talmon JL, Moorman PW, Kors JA. Mapping the domain of medical informatics. Methods Inf Med. 2009; 48: 76-83.

12. Yergens DW, Tam-Tham H, Minty EP. Visualization of the IMIA yearbook of medical informatics publications over the last 25 years. Yearb Med Inform. 2016; Suppl 1: S130-138.

13. Moen A, Knudsen LMM. Nursing informatics: Decades of contribution to health informatics. Healthc Inform Res. 2013; 19: 86-92.

14. Brennan PF, Bakken S. Nursing needs big data and big data needs nursing. J Nurs Scholar. 2015; 47: 477-484.

15. Bakken S, Reame N. The promise and potential perils of big data for advancing symptom management research in populations at risk for health disparities. Ann Rev Nurs Res. 2016; 34: 247-260.

16. Mantas J, Ammenwerth E, Demiris G, Hasman A, Haux R, Hersh W, et al. Recommendations of the International Medical Informatics Association (IMIA) on Education in Biomedical and Health Informatics. First Revision. Methods Inf Med. 2010; 49: 105-120.

17. Kulikowski CA, Shortliffe EH, Currie LM, Elkin PL, Hunter LE, Johnson TR, et al. AMIA Board wAMIA Board white paper: definition of biomedical informatics and specification of core competencies for graduate education in the discipline. J Am Med Inform Assoc. 2012; 19: 931-938.

18. Cornet R, Andersen SK, Stoicu-Tivadar L, Parra Calderón CL, Hercigonja-Szekeres M, Hörbst A, editors. Digital Healthcare Empowering Europeans: Proceedings of MIE2015. Amsterdam: IOS Press; 2015.

19. Sarkar IN, Georgiou A, Mazzoncini de Azevedo Marques, P, editors. MEDINFO 2015: eHealthenabled Health. Proceedings of the 15th World Congress on Health and Biomedical Informatics. Amsterdam: IOS Press; 2015

20. Hörbst A, Hackl WO, de Keizer N, Prokosch HU, Hercigonja-Szekeres M, de Lusignan S, editors. Exploring Complexity in Health: An Interdisciplinary Systems Approach. Proceedings of MIE2016 at HEC2016. Amsterdam: IOS Press; 2016.

21. Asia Pacific Association for Medical Informatics [cited 2016 Sept 25]. Available from: http://www. apami.org.

22. American Medical Informatics Association (with American College of Medical Informatics) [cited 2016 Sept 25]. Available from: https://www.amia. org.

23. Grémy F. Hardware, software, peopleware, subjectivity. A philosophical promenade. Methods Inf Med. 2005; 44: 352-358.

24. Ainsworth J, Buchan I. Combining health data uses to ignite health system learning. Methods Inf Med. 2015; 54: 479-487.

25. Detmer DE. At last! A working model of a data ecosystem for continuous learning in the evolving health noosphere. Methods Inf Med. 2015; 54: 477-478.

26. Denaxas S, Friedman CP, Geissbuhler A, Hemingway $\mathrm{H}$, Kalra D, Kimura M, et al. Discussion of "Combining health data uses to ignite health sys- tem learning". Methods Inf Med. 2015; 54: 488-499.

27. Ammenwerth E. Evidence-based health informatics: How do we know what we know? Methods Inf Med. 2015; 54: 298-307.

28. Rigby M. Optimising health informatics outcomes - getting good evidence to where it matters. Methods Inf Med. 2015; 54: 295-297.

29. Al-Shorbaji N, Hanmer L, Hussein R, Magrabi F Moen A, Moura LA, et al. Discussion of "Evidence-based health informatics: How do we know what we know?". Methods Inf Med. 2015; 54: 308-318.

30. Deserno TM, Marx N. Computational electrocardiography: revisiting Holter ECG monitoring. Methods Inf Med. 2016; 55: 305-311.

31. Yana K. Editorial for "Computational electrocardiography: revisiting Holter ECG monitoring". Methods Inf Med. 2016; 55: 303-304.

32. Baumgartner C, Caiani EG, Dickhaus H, Kulikowski CA, Schiecke K, van Bemmel JH, Witte H. Discussion of "Computational electrocardiography: revisiting Holter ECG monitoring”. Methods Inf Med. 2016; 55: 312-321.

33. Martin-Sanchez FJ, Lopez-Campos GH. The new role of biomedical informatics in the age of digital medicine. Methods Inf Med. 2016; 55, 392-402.

34. Shortliffe EH. Digital Medicine and Biomedical Informatics: What's in a Name? Methods Inf Med. 2016; 55, 389-391.

35. Al-Shorbaji N, Bellazzi R, Gonzalez Bernaldo de Quiros F, Koch S, Kulikowski CA, Lovell NH, et al. Discussion of "The new role of biomedical informatics in the age of digital medicine”. Methods Inf Med. 2016; 55, 403-421.

36. Correa A, Hinton W, McGovern A, van Vlymen J, Yonova I, Jones S, de Lusignan S. Royal College of General Practitioners Research and Surveillance Centre (RCGP RSC) sentinel network: a cohort profile. BMJ Open. 2016; 6: e011092.

37. de Lusignan S, van Weel C. The use of routinely collected computer data for research in primary care: opportunities and challenges. Fam Pract. 2006; 23: 253-263.

38. Chambers JC, Loh M, Lehne B, Drong A, Kriebel J, Motta V, et al. Epigenome-wide association of DNA methylation markers in peripheral blood from Indian Asians and Europeans with incident type 2 diabetes: a nested case-control study. Lancet Diabetes Endocrinol. 2015; 3: 526-534.

39. Kimura M, Shimizu K, Kuwahara M, Kaihara S, Koyama T, Tsuchiya F, et al. Knowledge-based antibiotic medication counselling system $\mathrm{AN}$ TICIPATOR and its implementation by Prolog. MEDINFO83. 1983; 589-592.

40. Fukuda S, Maeda A, Kimura M. Development of an expert system for welding design support (to provide advice on determination of weld condition to prevent weld cracking in a pressure vessel). J Jap Soc Mech Eng. 1986; 52: 1183-1190. (Japanese).

41. Nomura K, referring to Matsura Kiyoshi, "Kendan", 1821

42. Shortliffe EH, Perreault L, editors; Wiederhold G Fagan LM, associate editors. Medical informatics: Computer applications in health care and biomedicine. New York: Springer; 2000.

43. Neskey DM, Osman AA, Ow TJ, Katsonis P, McDonald T, Hicks SC, et al. Evolutionary action score of TP53 identifies high-risk mutations as- sociated with decreased survival and increased distant metastases in head and neck cancer. Cancer Res. 2015; 75: 1527-1536.

44. Hutcherson C, Bushong B, Rangle A. A neurocomputational model of altruistic choice and its implications. Neuron. 2015; 87: 451-462.

45. Strombach T, Weber B, Hangebrauk Z, Kenning P, Karipidis II, Tobler PN, Kalenscher T. Social discounting involves modulation of neural value signals by temporoparietal junction. PNAS. 2015; 112: 1619-1624.

46. Kuhn KA, Knoll A, Mewes HW, Schwaiger M, Bode A, Broy M, et al. Informatics and medicine from molecules to populations. Methods Inf Med. 2008; 47: 283-295.

47. Lakoff G, Nunez RE. Where mathematics comes from: How the embodied mind brings mathematics into being. New York: Basic Books; 2000.

48. Kulikowski CA, Kulikowski CW. Biomedical and health informatics in translational medicine. Methods Inf Med. 2009; 48: 4-10.

49. Ruiz-Calderon JF, Cavallin H, Song SJ, Novoselac A, Pericchi LR, Hernandez JN, et al. Walls talk: Microbial biogeography of homes spanning urbanization. Sci Adv. 2016; 2: e1501061.

50. Fauconnier G, Turner M. The Way We Think: Conceptual blending and the mind's hidden complexities. New York: Basic Books; 2002.

51. Payne PR Starren J. Quantifying visual similarity in clinical iconic graphics. J Am Med Inform Assoc. 2005; 12: 338-345.

52. Gray J. The Hilbert challenge. Oxford: Oxford University Press; 2001.

53. Haux R, Ammenwerth E, Herzog W, Knaup P. Health care in the information society. A prognosis for the year 2013. Int J Med Inform. 2002; 66: 3-21, discussion 23-120.

54. Martin Sanchez F, Gray K, Bellazzi R, Lopez-Campos G. Exposome informatics: Considerations for the design of future biomedical research information systems. J Am Med Inform Assoc. 2014; 21: 386-390.

55. Hamacher A, Kim SJ, Cho ST, Pardeshi S, Lee SH, Eun SJ, Whangbo TK. Application of virtual, augmented, and mixed reality to urology. Int Neurourol J. 2016; 20: 172-181.

56. Moen A, Knudsen LMM. Nursing informatics decades of contributions to health informatics. Healthcare Inf Res. 2013; 19: 86-92.

57. Ludwig von Bertalanffy - passages from "General System Theory” (1968) [cited 2016 Sept 25]. Available from: http://www.panarchy.org/vonberta lanffy/systems.1968.html.

58. Wikipedia - Shannon-Weaver model [cited 2016 Sept 25]. Available from: https://en.wikipedia.org/ wiki/Shannon\%E2\%80\%93Weaver_model.

59. Newell A, Simon HA. Human problem solving. Englewood Cliffs, NJ: Prentice-Hall; 1972.

60. Blois MS. Information and medicine: the nature of medical descriptions. Oakland, CA: University of California Press; 1984

61. Smith MJ, Liehr PR, editors. Middle range theory for nursing, 2nd ed. New York: Springer; 2008.

62. Coiera E. When conversation is better than computation. J Am Med Inform Assoc. 2000; 7: 277-286.

63. Collins SA, Bakken S, Vawdrey DK, Coiera E, Currie LM. Clinician preferences for verbal communi- 
cation compared to EHR documentation in the ICU. Appl Clin Inform. 2011; 2: 190-201.

64. Collins SA, Bakken S, Vawdrey DK, Coiera E, Currie LM. Agreement between common goals discussed and documented in the ICU. J Am Med Inform Assoc. 2011; 18: 45-60.

65. Collins SA, Mamykina L, Jordan D, Stein DM, Shine A, Reyfman P, Kaufman, K. In search of common ground in handoff documentation in an intensive care unit. J Biomed Inform. 2012; 45: 307-315.

66. de Lusignan S, Crawford L, Munro N. Creating and using real-world evidence to answer questions about clinical effectiveness. J Innov Health Inform. 2015; 22: 368-373.

67. Liyanage H, Liaw ST, Kuziemsky C, Di Iorio CT, Schreiber R, Terry AL, de Lusignan S. Building a privacy, ethics and data access framework for real world computerised medical record system data: A Delphi study. A Contribution of the IMIA Primary Health Care Informatics Working Group. Yearb Med Inform. 2016; 11: 11-18.

68. Ibáñez A, Larrañaga P, Bielza C. Predicting citation count of bioinformatics papers within four years of publication. Bioinform. 2009; 25: 3303-3309.

69. Currie LM. Evaluation frameworks for nursing informatics. Int J Med Inform. 2005; 74: 908-916.

70. Gray K, Sockolow P. Conceptual models in health informatics research: A literature review and suggestions for development. JMIR Med Inform. 2016; 4: e7.

71. Gruber TR. A translation approach to portable ontology specification. Knowledge Acquisition. 1993; 5: 199-220.

72. Noy NF, McGuinness DL. Ontology development 101: A guide to creating your first ontology. 2001 [cited 2016 Sept 25]. Available from: http://protege.stanford.edu/publications/ontology_development/ontology101-noy-mcguinness.html.

73. Pigott K, de Lusignan S, Rapley A, Robinson J, Pritchard-Copley A. An informatics benchmarking statement. Methods Inf Med. 2007; 46: 394-398.

74. de Lusignan $S$. What is primary care informatics? Am Med Inform Assoc. 2003; 10: 304-309.

75. Lakoff G. Explaining Embodied Cognition Results. Topics in Cognitive Science. 2012; 4: 773-785.
76. de Lusignan S, Krause P. The Hayes principles: learning from the national pilot of information technology and core generalisable theory in informatics. Inform Prim Care. 2010; 18: 73-77.

77. Abbasi K. Compulsory registration of clinical trials. BMJ. 2004; 329: 637-638.

78. Marsh AA, Stoycos SA, Brethel-Haurwitz KM, Robinson P, VanMeter JW, Cardinale EM. Neural and cognitive characteristics of extraordinary altruists. PNAS. 2014: 111: 15036-15041.

79. Hutcherson C, Montaser Kouhsari L, Woodward J, Rangel A. Emotional and utilitarian appraisals of moral dilemmas are encoded in separate areas and integrated in ventromedial prefrontal cortex. J Neurosc. 2015; 35: 12593-12605.

80. Pigott K, de Lusignan S, Rapley A, Robinson J, Pritchard-Copley A. An informatics benchmarking statement. Methods Inf Med. 2007; 46: 394-398.

81. Merton K. Social theory and social structure. New York: Free Press; 1968. 